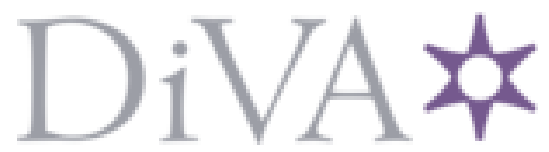

http://www.diva-portal.org

\title{
Postprint
}

This is the accepted version of a paper published in Contemporary Nurse: health care across the lifespan. This paper has been peer-reviewed but does not include the final publisher proof-corrections or journal pagination.

Citation for the original published paper (version of record):

Mattsson, K., Pietilä Rosendahl, S. (2017)

Teaching gerontology in globalized academics: a qualitative study of Thai nursing students' views on ageing when studying abroad..

Contemporary Nurse: health care across the lifespan, 53(1): 36-47

https://doi.org/10.1080/10376178.2016.1257921

Access to the published version may require subscription.

N.B. When citing this work, cite the original published paper.

Permanent link to this version:

http://urn.kb.se/resolve?urn=urn:nbn:se:esh:diva-5882 


\title{
Teaching gerontology in globalized academics: \\ A qualitative study of Thai nursing students' views on aging \\ when studying abroad
}

\author{
Authors: Karin Mattsson ${ }^{\mathrm{a}^{*} 1}$, Sirpa Pietilä Rosendahl ${ }^{\mathrm{a}}$ \\ ${ }^{a}$ School of Health, Care and Social Welfare, \\ Mälardalen University, Eskilstuna and Västerås, Sweden \\ *Correspondence: \\ Karin Mattsson, \\ School of Health, Care and Social Welfare, Mälardalen University \\ Box 325, SE-63105 Eskilstuna, Sweden \\ Mobile: +4670746 4243 Mail: karin.mattsson@mdh.se
}

\section{Acknowledgements and funding}

The authors would like to thank the international students who participated in this study. We also want to thank Joelle Caporaso and Dan Rosendahl for reviewing the manuscript. The Double Degree Bilingual Nursing Program (DDBNP) itself is funded by Mälardalen University, Sweden and Rangsit University, Thailand. The language revision and ethical vetting were funded by Mälardalen University, Sweden.

\section{Contribution}

Both authors were equally involved in all parts of this study; the planning, implementation, analysis and drafting of the manuscript.

\section{Conflict of interest}

None by any of the authors.

\footnotetext{
${ }^{1}$ Also affiliated to: Department of Health Care Sciences, Ersta Sköndal Community College, Stigbergsgatan 30, Box 111 89, SE-100 61 Stockholm, Sweden, Mail: karin.mattsson@esh.se
} 


\section{Teaching gerontology in globalized academics: A qualitative study of Thai nursing students' views on aging when studying abroad}

\section{Abstract}

Background: Negative views towards ageing and older adults may be a reason why nurses do not choose to work in gerontological nursing. Studying in another cultural context can challenge these views. The objective was to explore nursing students' views on ageing and older adults before and after a gerontology course held abroad. Design and method: A qualitative approach based on content analysis of responses to open-ended questions by 30 Thai nursing students studying a gerontology course in Sweden. Results: Three main categories; positive imprints of ageing, ageing takes its toll and knowledge leading to action, emerged through subcategories carrying a view of older adults as not only in need of care, but also as resourceful and competent. Professional health care, besides family was seen as potential caregivers in old age. Conclusions: Studying gerontology abroad can widen views towards ageing and older adults, inspiring nurses to work in gerontological nursing.

\section{Keywords}

Ageing, Culture, Gerontology, Nurse Education, Qualitative, Views 


\section{Teaching gerontology in globalized academics: A qualitative study of Thai nursing students' views on ageing when studying abroad}

\section{Background}

The elderly population is growing, and by 2050 , nearly four fifths of the world's older adults will be living in the less developed regions of the globe (UN, 2013). The increasing ageing population promises to exert pressure on all aspects of the health care continuum, from health promotion to disease prevention, long term care, and palliative care. There will also be an increasing demand for more nursing skills in assessing older persons' health and acute care needs in hospitals, and in primary care (Penney, Poulter, Cole \& Wellard, 2015). Besides, there is a growing need for registered nurses who are committed to working with older adults. Historically, a career in gerontological nursing has not been highly sought after, and earlier research indicates that one of the reasons for the lack of interest may be related to negative views towards older adults (Happell, 2002; Henderson, Xiao, Siegloff, Kelton, \& Paterson, 2008; McCann et al., 2010). Research has also highlighted the impact of education in gerontology on views towards working with older adults (Kydd et al., 2014; Liu et al., 2013).

A positive learning experience, supportive nurse educators, and focusing on healthy, normal ageing in the course content can challenge nursing students' views on aging, and influence nurses' career choices towards gerontological nursing (Carlsson \& Idvall, 2015; Runkawatt et al., 2013; Ferrario et al. 2008). Correspondingly, negative views towards older adults risk being cemented if the course content has an emphasis on morbidity, functional, and cognitive decline, and limitations in old age (Happell, 2002, Henderson et al., 2008). 
Until recently, the literature on gerontology, and views of ageing and older adults has mainly emphasized Western cultural contexts. One assumption is that negative attitudes toward the ageing processes and older adults are more common in the youth-obsessed Western nations (van den Heuvel, 2012), while the cultural values in Asian societies result in a more positive view of, and respect for older adults. Asian countries are known for their collectivistic culture, while the western part of the world and countries like Sweden are more individualistic. Older Swedes are typically living independent lives where municipalities are responsible for providing home care services for those who are living in ordinary housing. Instead of being dependent on help from families many older Swedes want to move to "senior housing" and residential aged care when the care needs are extended, and they no longer can cope with daily activities on their own (Granbom, 2014; Pietilä Rosendahl, Söderman \& Mazaheri, 2016). The Asian countries on the other hand, focuses on harmonious relationships and filial piety, and older adults share the same house as their children. Nevertheless, this is changing as fertility declines, and an ageing population alongside socioeconomic development threaten to bring about an erosion of the multigenerational family structure. Older adults may no longer be seen as deserving of respect and authority simply due to their age (Cheng et al, 2015b; Kim et al., 2015; Lin, 2015; Löckenhoff et al., 2009; Rittirong, 2014; WHO, 2015). Given the increase of the older population in e.g. Thailand, the formal health care system is facing new challenges. The demand for professionals having gerontological and geriatric competence will be increasing, and teaching gerontology during nursing education important. From a pedagogical point of view, what can be done to make working in gerontological nursing attractive for nurses in Thailand as well as in other parts of the world? 
Research on the impact of gerontological education on students' views of ageing and older people suggests it is important for nurse educators to include different views of ageing, as well as to present a holistic picture to the care of older adults that includes both healthy and pathological ageing processes (Kydd et al., 2014; Liu et al., 2013). Given the changing global demographics, where an increasingly older population is accompanied by culturally diverse societies, cross-cultural aspects on the care of older adults should be included within nursing education. With intercultural collaboration between universities, students studying abroad can be exposed to cultural contexts and values different than in the home country, which may contribute to mutual learning, and awareness of cultural diversity. One such co-productive project, is the Double Degree Bilingual Nursing Program (DDBNP) which takes place between two universities, one in Sweden and one in Thailand, where the Thai nursing students come to study in Sweden during their final year before graduation. This study focuses on how a gerontology course within the DDBNP program, that is, in another cultural context than in the students' home country, may influence the students. Therefore the aim of this study was to explore Thai nursing students' views towards ageing and older adults before and after a course in gerontology.

\section{Method}

A qualitative approach was chosen to explore and provide a deeper understanding of the Thai nursing students' views on ageing and older adults as they, in a reflective assignment, expressed their thoughts in their own words. The data was analysed using content analysis described by Graneheim and Lundman (2004) and Krippendorff (2004) which is a widely used 
research technique when studying a variety of subject matter. It is particularly suitable when analysing reflective narrative data in care science research and education.

\section{Study context}

Within the DDBNP in Sweden, the Thai nursing students are taking a five-week full time course in gerontology, which is collaboratively taught in English by Swedish and Thai lecturers. The course in gerontology is held during the students' last months of their year in Sweden. The course content covers cultural views on ageing, healthy and pathological ageing processes, and palliative care. The teaching about healthy ageing is equivalent to the focus on pathological ageing processes within gerontological nursing. Linking theory and empiricism together, the students in the course meet retired active older Swedish adults, interviewing them about their daily life in old age. Films, documentaries, seminars, and field studies at activity centres, day care facilities, senior housing, and residential aged care are also included in the course content.

\section{Participants}

The participants were 30 Thai nursing students (29 females/1 male) with an age range of 20-25 years (mean age 22.5 years). Before coming to Sweden the students' proficiency in English was assured. No one had previous working experience in old age care.

\section{Procedure}

The Thai nursing students were asked to write a reflection assignment with open-ended questions, where they reflected over their thoughts on ageing and on older adults. This was done on the first day, and on the last day of the course. The first three questions were the same 
before and after the course, the fourth additional question was asked after the course (see table $1)$.

\section{Table 1: Open-ended questions}

The above questions serve to differentiate between students' views on older adults, and old age as a state versus the process of ageing itself. The reflection assignment was mandatory, but the students were given the information that participation in the study was voluntary, and that they could withdraw from the study without having to explain the reasons. They were also assured that the material was confidential, and that no names would be included. Every student $(n=30)$ who were asked to participate agreed to do so.

\section{Data analysis}

The data was analysed using content analysis according to Graneheim and Lundman (2004) and Krippendorff (2004). The same analysis procedure was conducted on the data from the first and the last course date. The open-ended responses were analysed on a manifest level, where the visible, and obvious components of the text were described (Graneheim \& Lundman, 2004; Krippendorff, 2004). The data was first read through several times by both authors (KM, SPR) to obtain a sense of the whole. The second step included the re-reading of the data, in this step meaning units related to the aim of the study were identified. A meaning unit is a part of a sentence, or a complete sentence, or several sentences containing aspects related to each other and the aim of the study. The third step was to condense the meaning units; i.e. shortening the text while preserving the core meaning. In the fourth step meaning units were labelled with 
codes. In the fifth step codes that were similar were sorted into sub-categories, and in the sixth step, those with similar content were abstracted into main categories. Finally, the categories were separately checked and revised by the two authors for congruence with all of the steps in the analytical process, and the tentative categories were discussed until an agreement was reached about the final three main categories and six sub-categories.

\section{Ethical considerations}

The study was approved by the regional ethical review board at Uppsala University, Uppsala, Sweden (Dnr. 2015/322). Prior to the study, the participants received oral and written information about the purpose of the study, the voluntary nature of participation, and the question of confidentiality. It was stressed that the responses to the questionnaire and participation in the study did not affect the course results or grades in any way.

\section{Results}

The results show both positive and negative views on ageing and older adults. Three main categories and six sub-categories emerged from the analysis. The main categories were as follows: Positive imprints of ageing, Ageing takes its toll, and Knowledge leading to action, with the following sub-categories; wisdom through longevity, older adults are resourceful and competent, older adults lose appearance and functions, need for care and support, more than an ageing body, and wish for knowledge to take action.

Table 2: Main categories and subcategories 


\section{Positive imprints of ageing}

This first main category focuses on the positive aspects of ageing, and older adults being wise, resourceful and competent.

\section{Wisdom through longevity}

Already before the course the Thai students emphasized that older adults have developed wisdom and better judgement through their long life experience. Having lived through the many challenges of life, older adults can see the world differently than the young. Further, the process of ageing and longevity provides interesting life stories, those stories can serve to transfer knowledge from one generation to the next. The old can teach and give life guidance to the younger generations, in the eyes of the students.

Their [older adults'] experience of life can be an example and teach future generations. They are more often also in the position to know what to do when they are faced with problems. An older person always has a lot of interesting stories to tell me.

After the course the emphasis of older adults carrying wisdom, and transferring knowledge to the young was even greater. The stories older persons tell about their lives can teach the young about what is good and what is bad. The encounters with active Swedish older adults had made a positive impression on the students, ageing no longer appeared as horrible.

It is a challenge to learn a new experience in becoming old, but the older adults adjust and appreciate the good things in life more than the younger. 
In contrast to the young, older adults are more relaxed and have confidence from reaching their goals in life. They have free time and the ability to study; life-long learning is therefore possible. Old age can be the time for self-realization and dignity. By becoming of age a better judgment is formed, the older adults are the experts with coping experiences.

\section{Older adults are resourceful and competent}

Before the course the positive traits assigned to older adults included: nice, kind, cute and loveable as well as being competent, in the sense of knowing how to deal with challenges. When facing the challenges of old age, older adults can adjust their daily activities and lifestyle to their changing mental and physical abilities. This requires acceptance, which also was highlighted by the students; if the older adults have accepted being old, the results of the ageing processes are easier to handle and prepare for. One Thai student described her experience of her encounters with the Swedish older seniors:

I honestly admit my first thought of becoming old is suffering. However, the opportunity to talk with active [Swedish] older adults [during the course] brought me into another world of healthy ageing and declining happily.

After the course the students reflected upon the resources the older adults had gained through life. Older adults seem to be aware of self-care activities that can promote physical as well as psychological and spiritual health. This was surprising and reassuring for the students. The students reflected upon how the older adults are happier with their lives than they had 
expected; the older adults appeared to be more content now than they were in their youth. One student commented:

Ageing, it's not a bad thing; the old people that I met during this course seem to be very happy, they continue doing a lot of activities, learning new things and enjoying their life as much as they can.

The Thai students stressed that even if the Swedish older adults appeared frail with a declining physical condition, the Swedish seniors' attitude and thinking were young, and they were motivated to live long with a good quality of life. To live until old age demands motivation and inspiration; therefore, older adults are those who have learned most.

\section{Ageing takes its toll}

However, the views varied and the second category focuses on the negative aspects of ageing. The students described older adults as frail and in need of care and support.

\section{Older adults lose appearance and functions}

Before the course negative traits attributed to older adults included that they are pitiful, clumsy, slow, and boring, as well as that they have poor hygiene, mood swings, and lose control of themselves. The physical expressions of the ageing process, such as the disintegration of skin, muscles, hair, and attractiveness are viewed negatively, and the students perceived that older adults experience stress due to their changed body image. All of the Thai students made 
statements that revealed a view of seeing older adults as frail due to experiencing physical changes, loss of function and multiple diseases. This was explicitly expressed with words that displayed a training in pathology, with students mentioning complicated diseases like DM, hypertension, heart disease, UTI, cognitive impairment, pain, paralysis, stroke, Parkinson's, loss of memory, hearing, vision, developing dementia and having sexual disability. The students focus on beauty, and loss of physical appearance in aging, was apparent, especially before the course. After the course this was not mentioned in the same extent and one student express the change:

Before I wanted to be beautiful, I wanted plastic surgery, inject some chemical to make me look young. But now I think I want to live as long as I can without painful procedures. All of my thinking about cosmetic surgery has been removed of my mind.

However, the students did not consider ageing itself a disease but a process of decline, and various losses that sometimes leads to a loss of hope. Those that are ageing are running out of life and have nothing to live for. The Thai students reflected on the fact that older persons will meet death soon, and that the death of others is always present. After the course the students expressed that they view older adults as frail in reference to their physical condition and function, but they also realize there is more to it than caring for the body; social interaction, mental stimulation and assistance is equally important. One student shared:

I now have more understanding with them and I know more about specific nursing care for the older adult with dementia, like using music and touch in dementia therapy. With 
chronic illness, we should promote quality of life and have a [palliative] approach including prevention and relief of physical, psychological, psychosocial and spiritual suffering.

\section{Need for care and support}

Before the course the need for care and support were stressed in that the older adults need someone to take care of their health. They are dependent not only on their family but also on professional caregivers to stay healthy. Another reason for giving care and support to older adults was related to social aspects. Ageing was connected by the Thai students to loneliness, dependence and on being a burden to the family. One of the students described:

They stay alone and don't have help from a caregiver and relatives because relatives work a lot. They should have a person beside them such as a family member or caregiver to care for them and prevent accidents from happening.

The importance of family taking care of the older adults was emphasized and values were transferred through the family environment. The Thai nursing students themselves had made the majority of their own observations of ageing and older people based on relationships with older family members. The students saw themselves caring for family members in the future, as well as being old themselves, and having to be cared for by children and grandchildren. After the course the students had learned that there are differences between how older people in Thailand and Sweden look upon their need of care and from whom they want it. Older adults 
can actually have good quality of life in senior housing, where they don't need to live together with their adult children. A student reflected:

I still think the same about frailty of the body, but it amazed me how active they were in their lives. Moreover, I found that family members are important also in Sweden, but with illness they want professionals to support and empower them. The knowledge of the health care provider is important because most of elderly have some disease and health problem. And the communication between health care provider and older people is very important too.

\section{Knowledge leading to action}

Views related to knowledge of ageing and older adults inspired the Thai nursing students to think about how they should apply the knowledge, as seen in this third main category.

\section{More than an ageing body}

Both before and after the course the Thai students emphasized that older persons are deserving of respect, and that the young show this. The older adults earn respect both according to Thai culture, and from their long experience with life. Although respect for the older generations is a cultural trait in Thailand, the value of the word "respect" after the course seemed to be more diverse. The students then focused less on pathology and the ageing body; rather, the social, psychological, and existential aspects of ageing were emphasized more than before the course. One student described: 
[As a nurse] I have to take care not only of the body but also of the mental, and emotional health [of patients], which are important.

At the same time as the Thai students reflected on ageing, and older adults from a more holistic view point, they also seemed to move from a collective to an individualistic perspective on old age care as shared by one of the students below.

They are individuals; some have hearing problems or some have cognitive impairment, so the important point is that we concern ourselves with the individual conditions and try to find the best way of dealing with them and serving them well.

The students also seemed to have realized that living in a Swedish senior housing does not have to imply low status and being abandoned by the family. It was actually preferred by some of the older Swedish adults.

\section{Wish for knowledge to take action}

After the course the students reflected upon how they have to take action to improve the quality of life for the older adults. Improved quality of life was considered attainable through listening and communicating with older adults about their care, as well as improving knowledge of ageing to distinguish when, and if a condition is curable. The students focused on the need for more knowledge about both normal and pathological ageing because they, as nurses, can enhance the quality of life and wellness of the older adults. 
The Swedish context was for the Thai nursing students different in terms of language, social and cultural values. Including cultural perspectives were noted as being important for the basic understanding of ageing, and how to care for older adults. Some were inspired to work with older adults. One student commented:

The course has given me knowledge about different cultures, and the differences between older adults in Thailand and in Sweden. This is important when taking care of an older adult as a nurse. I will try to blend cultures together, and take care of them with dignity. I plan to learn more about gerontology in the future.

The course and the encounters with older Swedish adults in class and during field studies, appear to have met the challenges of some of the students' own existential thoughts, and made them think about their own ageing. One student concluded:

I am now admiring those who are older adults, having life expertise and life success. Becoming old is not something I need to be afraid of anymore. Sometimes I can understand the future of me.

When summarizing the categories, the first category showed a picture of the older adult as wise and experienced, which was almost the same before and after the course. The aspect of the older adults as competent and resourceful was added to the picture after the course, maybe due to the encounters with active Swedish older adults. The total picture of the older adult was not entirely positive, as seen in the second category, which pointed to the older adult also as frail 
due to the losses in old age, and therefore in need of care. What was changed after the course in this category was that the nursing students also saw others, outside the family as possible caretakers. In the third category the students expressed that actions to improve quality of life were needed, and that they as nurses could make use of their acquired knowledge in gerontology. Some were also inspired to work with older adults.

\section{Discussion}

This study aimed to explore Thai nursing students' views towards ageing and older adults in connection with a course in gerontology held in Sweden. The results indicate that the course influenced the students on different levels. One such level appears to be a diverse view of older adults as not only people in need of care and support, but also as competent and able to manage life in a good way. This viewpoint may be attributable to the encounters with the Swedish seniors that visited the class and spoke about their lives. These encounters, and talking to older adults in residential care who were happy, despite multi-morbidity (including dementia) and not living with their families, made an impression on the students. This is consistent with studies by Ferrario et al., (2008) and Wilkinson et al., (2002), pointing to the benefits of studying gerontology through meetings with older adults, and to Osuji (2014), whose work recommends one-on-one mentoring of nursing students by older adults. When students are exposed to older populations in all phases of the health care continuum, their gerontological knowledge and positive attitudes towards older adults will expand, increasing their competency level in practice. Another level according to the results, was that the encounters with the Swedish seniors affected the students' views of the ageing process itself. It no longer appeared as frightening as before. To some it actually meant becoming aware of the positive sides of 
their own future, which is consistent with the study by Kalish, Coughlin, Ballard and Lamson (2013). Intergenerational learning, or where older adults and young students meet to engage in mutual learning, has been studied by Tam (2014), and was proven to be an effective way to engage the young and old in a manner that was beneficial to all participants involved. Apart from the benefits that were mainly relationship-based, the students were ultimately able to apply many of the academic concepts, theories and knowledge gleaned from the encounters to real-life situations. Additionally, the Thai students seemed to have gained a deeper knowledge and understanding of the process of ageing, particularly that of healthy/normal ageing versus unhealthy ageing, which may have to do with theoretical course content focusing equally on both. Concern and apprehension was expressed about adverse physical, mental and personal losses during the ageing process. The students' previous schooling in pathology could clearly be seen, as ageing was first and foremost connected with having multiple physical and psychological illnesses associated with functional decline. According to Rattan (2014) the understanding of biological ageing, either as a disease or as a process that increases the risk of disease onset, has serious implications with respect to interventional strategies. If ageing is considered a disease, then it can be argued that a disease-free state could be achieved with treatment. Thus, chronic, age-related illnesses imply a failure, which risks the abandonment of the older adult, and the spreading of ageism. Knowing the difference between healthy and pathological ageing is of utmost importance for nurses. The use of more diverse and positive language about ageing, as well as having a health-oriented approach, can change the way that health care workers treat and look upon older adults, with the same applying to society at large. Importantly, the negative words used describing older adults were complimented with words 
that indicated older adults are competent and wise. Runkawatt et al. (2013) emphasize that positive attitudes towards older people are a foundation for future nursing care.

Due to globalization, more people are moving far away from their country of origin either as refugees or voluntary migrants. This implies a growing amount of older immigrants, which poses a unique challenge for the healthcare and social service systems in the countries being settled (Kristiansen et al., 2015; Torres et al., 2016). Studying abroad in another cultural context, like the Thai nursing DDBNP students, seemed not only have had an impact on their views of ageing and older adults, but contributed to cultural awareness, and possible cultural competency. Thereby this study implicates that our practice can be generalizable in other cultural or geographic regions.

Teaching gerontology in nursing education, including various cultural aspects, will be crucial to meeting the challenges and new opportunities that may occur through changes in demographics. An awareness about the normality in aging, knowledge of natural aging processes as well as what is culturally important and relevant in service delivery, which has been acknowledged by Elliot (2005), may help change negative attitudes towards working within elder care (Engström \& Fagerberg, 2011; McCann et al., 2010). The demand for education in gerontology and geriatrics is now also emerging from research within Australasia and Africa (Cheng et al., 2015a; Fajemilehin, 2004; Henderson et al., 2008; Hirst \& Cole, 2014; Kumar et al., 2012; Liu et al., 2013), stressing that the amount of education in these areas will have an impact on the students' understanding of the needs of elderly patients (Fagerberg \& Gilje, 2007).

For nurses, whose main focus is on ensuring the bodily needs, care and treatment of people with various medical conditions, increased knowledge of the natural ageing processes is 
crucial to be able to distinguish when, and if a condition is possible to cure. Nevertheless, knowledge of physical needs is not enough, given that normal ageing includes social, psychological and existential aspects that must be addressed and taken into consideration. In this study, the desire and quest for more knowledge were apparent in the students' reflections. This desire for more knowledge makes courses in gerontology a major concern for nursing education and confirms the results of other studies (e.g. Ferrario et al., 2008). These studies stress that the inclusion of positive aspects of ageing in curriculums may foster a paradigm shift, making students more likely to choose to work with older adults as part of their own life trajectories.

\section{Methodological considerations}

Instead of using quantitative methods and measures, this study used a qualitative approach to explore how the students would respond when given the opportunity to narrate their answers to open-ended questions. A limitation here is that even though the questions are open-ended, the questionnaire can still be rather rigid in contrast to face-to-face interviews where the interviewer can ask further questions to clarify unclear content. However, the variation obtained from writing long and short answers could partially compensate for any lack of depth of the material, and the amount of participants $(n=30)$ would also add to variation of the data as is requested in qualitative studies. A limitation regarding the analysis was that, when analysing rather short answers, the context surrounding the meaning units was sparse, making it somewhat difficult to interpret the data. Conversely, when the authors discussed the categories together, a mutual understanding and an agreement was reached. 


\section{Conclusion}

Studying gerontology within a nursing program abroad seems to contribute to widened views towards ageing and older adults. Older adults are not only seen as individuals in need of care, but also as competent and resourceful, and that family do not need to be their prioritized carers. This is important for an individualized care of older adults in a multi-cultural society. The need for nurses with gerontological knowledge being active in securing quality of life for older adults is extensive in all parts of the world. Teaching gerontology as a joint mission within internationalization projects of nursing education and globalized academics is a possibility to maximize reciprocal learning, and an interest in gerontological nursing.

\section{Acknowledgements and funding}

The authors would like to thank the international students who participated in this study. We also want to thank Joelle Caporaso and Dan Rosendahl for reviewing the manuscript. The Double Degree Bilingual Nursing Program (DDBNP) itself is funded by Mälardalen University, Sweden and Rangsit University, Thailand. The language revision and ethical vetting were funded by Mälardalen University, Sweden.

\section{Contribution}

Both authors were equally involved in all parts of this study; the planning, implementation, analysis and drafting of the manuscript.

\section{Conflict of interest}

None by any of the authors. 


\section{References}

Carlsson, E., \& Idvall, E. (2015). Who wants to work with older people? Swedish student nurses' willingness to work in elderly care - A questionnaire study. Nurse Education Today, 35, 849-853. DOI: 10.1016/j.nedt.2015.03.002

Cheng, M., Cheng, C., Tian, Y., \& Fan, X. (2015a). Student nurses' motivation to choose gerontological nursing as a career in China: A survey study. Nurse Education Today, 35, 843-848. DOI: 10.1016/j.nedt.2015.03.001

Cheng, S.T., Fung, H.H. Li, L.W., Li, T., Woo, J., \& Chi, I. (2015b). Successful aging: concepts, reflections and its relevance to Asia. In S.T. Cheng, I. Chi, H.H. Fung, L.W. Li, \& J. Woo. Successful Aging. Asian Perspectives. Heidelberg, New York, London: Springer.

Elliott, K.S. (2005). Incorporating cross-cultural perspectives that challenge assumptions about assessment and service delivery. Gerontology \& Geriatrics Education, 26(1), 23-41. DOI: 10.1300/J021v26n01_03

Engström, G., \& Fagerberg, I. (2011). Attitudes towards older people among Swedish health care students and health care professionals working in elder care. Nursing Reports, 1(4), 1520. DOI: $10.4081 /$ nursrep.2011.e2

Fagerberg, I., \& Gilje, F. (2007). A comparison of curricular approaches of care of the aged in Swedish and US nursing programs. Nurse Education Practitioner, 7(6), 358-364. DOI: 10.1016/j.nepr.2006.11.007

Fajemilehin, B. R. (2004). Attitudes of students in health professions toward caring for older people: Needed curricula revisions in Nigeria. Educational Gerontology, 30, 383-390. DOI: $10.1080 / 03601270490433573$ 
Ferrario, C., Freeman, F., Nellett, G., \& Scheel, J. (2008). Changing nursing students' attitudes about aging: An argument for the successful aging paradigm. Educational Gerontology, 34, 51-66. DOI: 10.1080/03601270701763969

Granbom, M. (2014). Relocation and residential reasoning in very old age - Housing, health and everyday life. Doctoral dissertation: Lund University. Retrieved from: file://C:/Users/kmn02/Downloads/Kappa_fAPr_elektronisk_spikning.pdf

Graneheim, U.H., \& Lundman, B. (2004). Qualitative content analysis in nursing research: Concepts, procedures and measures to achieve trustworthiness. Nurse Education Today, 24(2), 105-112. DOI: 10.1016/j.nedt.2003.10.001

Happell, B. (2002). Nursing home employment for nursing students: Valuable experience or a harsh deterrent? Journal of Advanced Nursing, 39(6), 529-536. DOI: 10.1046/j.13652648.2002.02321.x

Henderson, J., Xiao, L., Siegloff, L., Kelton, M., \& Paterson, J. (2008). 'Older people have lived their lives': First year nursing students' attitudes towards older people. Contemporary Nurse, 30(1), 32-45. DOI: 10.5172/conu.673.30.1.32

van den Heuvel, W. (2012). Discrimination against older people. Reviews in Clinical Gerontology, 22, 293-300. DOI: 10.1017/S095925981200010X

Hirst, S., \& Cole, M. (2014). The process of gerontological competence in the delivery of acute nursing Care. Indian Journal of Gerontology, 28(4), 456-468.

Kalish, H., Coughlin, D., Ballard, S. \& Lamson, A. (2013). Old age is a part of living: Student reflections on intergenerational service learning. Gerontology \& Geriatrics Education, 34:

99-113. DOI: 10.1080/02701960.2012.753440 
Kim, K., Cheng, Y-P., Zarit, S., \& Fingerman, K. (2015). Relationships between adults and parents in Asia. In S.T. Cheng, I. Chi, H.H. Fung, L.W. Li, \& J. Woo. Successful Aging. Asian Perspectives. Heidelberg, New York, London: Springer.

Krippendorff, K. (2004). Content Analysis. An Introduction to its Methodology. (2 ${ }^{\text {nd }}$ ed.). London, UK: Sage.

Kristiansen, M., Lue Kessing, L., Norredam, M., \& Krasnik, A. (2015). Migrants' perceptions of aging in Denmark and attitudes towards remigration: results from a qualitative study. BMC Health Services Research, 15, 225. DOI: 10.1186/s12913-015-0901-5

Kumar, A., Johnson, S., \& Kamalanabhan, T.J. (2012). The future of health care: Students' perceptions, education, and training in aging and health in India. Asia-Pacific Journal of Public Health, 24(6), 923-931. DOI: 10.1177/1010539511410686

Kydd, A., Engström, G., Touhy, T., Newman, D., Skela, B., Hvalic Touzery, S., Zurc, J., Galatsch, M., Ito, M., \& Fagerberg, I. (2014). Attitudes of nurses, and student nurses towards working with older people and to gerontological nursing as a career in Germany, Scotland, Slovenia, Sweden, Japan and the United States. International Journal of Nursing Education, 6(2), 191-199. DOI: 10.5958/0974-9357.2014.00630.8

Lin, J-P. (2015). Life satisfaction among older adults in Taiwan: The effects of marital relations and intergenerational relations. In S.T. Cheng, I. Chi, H.H. Fung, L.W. Li, \& J. Woo. Successful Aging. Asian Perspectives. Heidelberg, New York, London: Springer.

Liu, Y., Norman, I., \& While, A. (2013). Nurses' attitudes towards older people: A systematic review. International Journal of Nursing Studies, 50, 1271-1282. DOI:

10.1016/j.ijnurstu.2012.11.021 
Löckenhoff, C.E., De Fruyt, F., Terracciano, A., McCrae, R.R., De Bolle, M., Costa, P.T., et al. (2009). Perceptions of aging across 26 cultures and their culture-level associates. Psychology and Aging, 24(4), 941-954. DOI: 10.1037/a0016901

McCann, T.V., Clark, E., \& Lu, S. (2010). Bachelor of Nursing students career choices: A three-year longitudinal study. Nurse Education Today, 30(1), 31-36. DOI: 10.1016/j.nedt.2009.05.014

Osuji, J. (2014). Building global capacity for better health through increased gerontology content in undergraduate nursing education curriculum: A Commentary. Indian Journal of Gerontology, 28(4), 482-493.

Penney, W., Poulter, N., Cole, C. \& Wellard, S. (2015). Nursing assessment of older people who are in hospital: Exploring registered nurses' understanding of their assessment skills. Contemporary Nurse. Accepted author version posted online: 22 Oct 2015, 1-13. DOI: $10.1080 / 10376178.2015 .1111152$

Pietilä Rosendahl, S., Söderman, M. \& Mazaheri, M. (2016). Immigrant with dementia in Swedish residential care: An exploratory study of the experiences of their family members and Nursing staff. BMC Geriatrics, 16(18), 1-12. DOI: 10.1186/s12877-016-0200-y

Rattan, S. (2014). Aging is not a disease: Implications for intervention. Aging and Disease, 5(3), 196-202. DOI: 10.14336/AD.2014.0500196

Rittirong, J., Prasartkul, P., \& Rindfuss, R. (2014). From who do older persons prefer support? The case of rural Thailand. Journal of Aging Studies, 31, 171-181. DOI:

10.1016/j.jaging.2014.10.002 
Runkawatt, V., Gustafsson, C., \& Engström, G. (2013). Different cultures but similar positive attitudes: A comparison between Thai and Swedish nursing students' attitudes toward older people. Educational Gerontology, 39, 92-102. DOI: 10.1080/03601277.2012.689934

Tam, M. (2014). Intergenerational service learning between the old and the young: What, why and how. Educational Gerontology, 40, 401-413. DOI: 10.1080/03601277.2013.822201

Torres, S., Ågård, P., \& Milberg, A. (2016). The 'other' in end-of-life care: Providers' understanding of patients with migrant backgrounds. Journal of Intercultural Studies, 37(2), 103-117. DOI: 10.1080/07256868.2016.1141756

UN, United Nations. (2013). World Population Aging. Retrieved from http://www.un.org/esa/population/publications/worldageing19502050/

WHO, World Health Organization. (2015). Older Population and Health System: A profile of Thailand. Retrieved from http://www.who.int/ageing/projects/intra/phase_one/alc_intra1_cp_thailand.pdf Wilkinson, T.J., Gower, S., \& Sainsbury, R. (2002). The earlier, the better: The effect of early community contact on the attitudes of medical students to older people. Medical Education, 36(6), 540-542. DOI: 10.1046/j.1365-2923.2002.01226.x 
Teaching gerontology in globalized academics: A qualitative study of Thai nursing students' views on aging when studying abroad

Table 1

Open-ended questions

Write down the words you spontaneously associate with the words older, ageing and old"

What do you think has influenced your views and perceptions of being old/becoming old?"

What do you think when you meet a very old person?"

Describe in what way you think differently (or the same) of older adults after studying in this course". 


\section{TABLE 2}

Teaching gerontology in globalized academics: A qualitative study of Thai nursing students' views on aging when studying abroad

Table 2

Main/Subcategories

Main categories Subcategories

Positive imprints of ageing Wisdom through longevity

Older adults are resourceful and competent

Ageing takes its toll

Older adults lose appearance and functions

Need for care and support

Knowledge leading to action More than an aging body

Wish for knowledge to take action 